\title{
Could Molnupiravir Reduce Viral Burden of COVID-19?
}

\author{
Masoud Mardani (ii) ${ }^{1, *}$ \\ 1. Infectious Diseases and Tropical Medicine Research Center, Shahid Beheshti University of Medical Sciences, Tehran, Iran \\ "Corresponding author: 1. Infectious Diseases and Tropical Medicine Research Center, Shahid Beheshti University of Medical Sciences, Tehran, Iran Email: \\ drmasoudmardani@yahoo.com \\ Received 2021 September 26; Accepted 2021 September 26.
}

Keywords: COVID-19, Molnupiravir, Hospitalization

After over a year passing from the coronavirus disease 2019 (COVID-19) pandemic, global vaccination will help us end the COVID-19 pandemic. Although many drugs have been used to treat COVID-19 patients, researchers are still looking to detect antiviral agents with effectiveness on COVID-19 caused by the severe acute respiratory syndrome coronavirus 2 (SARS-CoV-2) (1).

Molnupiravir was reported by investigators of the University of North Carolina, Chapel Hill as the first directacting and oral antiviral to reduce the levels of viral ribonucleic acid (RNA) and nasopharyngeal SARS-CoV-2 infectious virus (1).

It has been shown that that molnupiravir is safe and well tolerated, making it an appropriate antiviral against SARS-CoV-2 (2).

So far, more than 200 million people have been infected by SARS-CoV-2 and 4 million people have died worldwide. This infection can cause severe symptoms in vulnerable people, including the elderly and cases with comorbidities (2).

There is an association between elevated SARS-CoV-2 nasopharyngeal RNA concentrations and the rate of hospitalization. Studies on animals have shown a link between viral RNA concentrations and transmission rate, as well (1).

There is no treatment to cure SARS-CoV-2and prevent the transmission of virus to other people. Researchers are racing for finding an appropriate medication, such as an oral antiviral, for reducing disease progression and avoiding transmission (1).

Preclinical investigations have declared molnupiravir wide range of antiviral activity against different coronaviruses, such as SARS-CoV-2. In humanized mouse models, using molnupiravir as a prophylaxis and treatment reduced viral replication and pathogenesis (1).

Molnupiravir is currently a drug candidate in phase III trials to treat COVID-19 patients. It enhances the rate muta- tions in viral RNA and can impair the replication of SARSCoV-2 in humans and animals. We established the molecular mechanisms of molnupiravir-related RNA mutagenesis using the viral RNA-dependent RNA polymerase (RdRp). According to biochemical assessments, the RdRp can use the molnupiravir active form as a substrate instead of uridine triphosphate or cytidine triphosphate. Following the use of the obtained RNA by RdRp as a template, $\mathrm{NHC}$ can direct incorporation of A or G, resulting in mutated RNA products. According to the structural assessment of RdRp-RNA complexes containing mutagenesis products, NHC forms constant base pairs with A or $\mathrm{G}$ in the active center of RdRp, which explains the way the polymerase can escape proofreading and synthesize mutated RNA. Such two-step mutagenesis mechanism is used in many viral polymerases, explaining the molnupiravir broad-spectrum antiviral activity (3).

Molnupiravir is an orally active experimental antiviral agent, which was developed to treat influenza by Merck (MSD), and Ridgeback Biotherapeutics.. It is currently assessed in Phase III clinical trial, the MOVe-OUT study, for the treatment of non-hospitalized cases with confirmed COVID-19 (4).

Outpatients with confirmed COVID-19 as well as symptom onset within seven days were included. The subjects randomly received 1:1 to molnupiravir $(200 \mathrm{mg}$ ) or placebo or 3:1 to molnupiravir ( 400 or $800 \mathrm{mg}$ ) or placebo. The medication was administrated orally two times a day for five days, with dose escalations upon the completion of the safety data from the present and other studies (1).

A total of 202 cases were studied and were administrated with at least one dose of placebo or molnupiravir at ten areas in the US, of whom seven cases did not continue the study because of adverse effects and withdrawal (1).

At baseline, the infectious viruses was isolated from $43.5 \%$ of nasopharyngeal swab samples. On day three, col- 
lected infections reduced to $1.9 \%$ in cases to whom molnupiravir was given at $800 \mathrm{mg}$, comparable to $16.7 \%$ of cases receiving the placebo (1).

Furthermore, infectious virus isolation also reduced on day five in cases who received molnupiravir (400 or $800 \mathrm{mg}$ ). No contagious virus levels were found in both groups, which is comparable to $11.1 \%$ of cases who received a placebo (1).

Regarding tolerability and safety, molnupiravir was showed few and low-grade adverse effects. The prevalence of adverse events caused by treatment was lowest in the group receiving the dose of $800 \mathrm{mg}$. The main adverse manifestations were elevated alanine aminotransferase levels, insomnia, and headache (1).

The trial findings demonstrated the antiviral effectiveness tolerability, and safety, of molnupiravir for reducing the replication of SARS-CoV-2 and accelerating clearance of infectious virus and supported ongoing trials of molnupiravir for preventing the COVID-19 progression and eliminating onward SARS-CoV-2 transmission.

In Iran due to increase COVID cases in recent months and he occupation of ICU beds and the abundant need for health services in hospitals, The initiation of antiviral drug in patient with acute Fever can lead to reduction of burden of the virus and the need for hospitalization and control of outbreaks.

\section{Footnotes}

Conflict of Interests: There is no conflict of interest. Funding/Support: No funding .

\section{References}

1. Angela Betsaida B L. An oral pill for COVID-19? Molnupiravir shows promise. 2021, [cited Jun 27 2021]. Available from: https://www.news-medical.net/news/20210627/An-oral-pillfor-COVID19-Molnupiravir-shows-promise.aspx.

2. WHO. novel-coronavirus 2019. 2019. Available from: https://www. who.int/emergencies/diseases/novel-coronavirus2019?gclid= EAIaIQobChMI8oaWnqOd8wIV2YbVCh0RcwAOEAAYASAAEgJkePD_ BwE.

3. Kabinger F, Stiller C, Schmitzova J, Dienemann C, Kokic G, Hillen HS, et al. Mechanism of molnupiravir-induced SARS-CoV-2 mutagenesis. Nat Struct Mol Biol. 2021;28(9):740-6. doi: 10.1038/s41594-021-00651-0. [PubMed: 34381216]. [PubMed Central: PMC8437801].

4. Clinical trials arena. Merck and Ridgeback begin Phase III trial of molnupiravir for Covid-19. Clinical trials arena; 2021. Available from: https://www.clinicaltrialsarena.com/news/merck-ridgebackmolnupiravir-trial/. 